\title{
Combinación de estrategias de innovación docente para la enseñanza de asignaturas de Ciencias de la Tierra: aprendizaje colaborativo y basado en proyectos como fuente de material para el aula invertida Combination of various innovation strategies for teaching Earth Sciences subjects: project-based and collaborative learning as source of material for the flipped classroom
}

\author{
Escavy, J.I. ${ }^{1}$, Sanz-Pérez, E. ${ }^{1}$, Menéndez-Pidal, I. ${ }^{1}$, Galindo-Aires, R. ${ }^{1}$, Fernández-González, E. ${ }^{1}$, Herrero, M.J. ${ }^{2}$, \\ Escudero, D. ${ }^{1}$, Trigos, L. ${ }^{1}$, Martínez-López, A. ${ }^{1}$, Sanz de Ojeda, J. ${ }^{1}$ \\ ji.escavy@upm.es, eugenio.sanz@upm.es, ignacio.menendezpidal@upm.es, rubenangel.galindo@upm.es, \\ eduardo.fgonzalez@alumnos.upm.es, mjherrer@ucm.es, diego.escudero@upm.es, laura.trigos@upm.es, \\ amparo.martinez@upm.es, joaquin.sanzydeojeda@gmail.com
}

${ }^{1}$ Dpto. de Ingeniería y Morfología del Terreno

Universidad Politécnica de Madrid Madrid, España

\author{
${ }^{2}$ Dpto. de Mineralogía y Petrología \\ Universidad Complutense de Madrid \\ Madrid, España
}

\begin{abstract}
Resumen- Se han combinado diferentes estrategias de innovación docente para la creación de material de uso en el aula invertida. Grupos de alumnos han realizado micro-videotutoriales para la resolución de problemas básicos de cortes geológicos, realizando ellos mismos desde el guion hasta la edición, pasando por la grabación. Mediante esta actividad han tenido que desarrollar un proyecto y trabajar de forma colaborativa. El resultado de este trabajo ha sido una colección de microvideos que están siendo empleados en la formación de otros alumnos mediante el aula invertida en varias asignaturas de Ciencias de la Tierra. Los videos se han puesto a disposición de los alumnos a través de la plataforma Moodle de la UPM para que puedan aprender y repasar las técnicas de resolución de problemas de forma autónoma.
\end{abstract}

Palabras clave: Microlearning, Aula Invertida, Aprendizaje Colaborativo, Aprendizaje basado en proyectos.

\begin{abstract}
Different teaching innovation strategies have been combined to create material to be used in the flipped classroom. Teams of students have made micro-videotutorials of how to solve basic problems of geological sections interpretation, from the script to the editing through the recording. By following this activity the students had to develop a project and work collaboratively. The result of this work is a collection of micro-videos that are used in flipped classroom training for Earth Sciences subjects. The videos have been made available for students through the UPM Moodle platform, and they can learn and review the troubleshooting techniques autonomously.
\end{abstract}

Keywords: Microlearning, Flipped Classroom, Collaborative Learning, Project-basedLearning.

\section{INTRODUCCIÓN}

La innovación docente en el entorno universitario viene desarrollando diferentes líneas desde hace varios decenios. Sin embargo, en España se han producido dos puntos de inflexión importantes. El primero fue con la adaptación al Espacio Europeo de Educación Superior (Plan Bolonia) en el que de forma proactiva las diferentes entidades han ido incorporando diferentes modelos pedagógicos como el aula invertida, el aprendizaje basado en proyectos y el aprendizaje colaborativo entre otros. El segundo gran salto se ha producido como una reacción para intentar adaptarse a la situación anómala provocada por la pandemia del COVID-19, momento en el que la tecnología ha soportado gran parte de los cambios de la metodología docente. En un tiempo record se han tenido que adaptar las metodologías docentes para migrar al ambiente virtual (Herrero et al., 2021, Moreno-Correa, 2020). En este contexto se han tenido que adaptar contenidos, metodologías, introducir herramientas de videoconferencia, evaluación, etc. que ha permitido realizar las clases en épocas de confinamiento y garantizar la distancia social en periodos con menores restricciones.

El modelo de aprendizaje del aula invertida consiste en intercambiar los roles y momentos de la enseñanza clásica. Para ello el estudiante se prepara la parte teórica de forma autónoma, con ayuda multimedia, reservando las horas de clase para las actividades prácticas y de aplicación de los conceptos aprendidos fuera del aula (Domínguez et al. 2015, MartínezOlvera, 2014, Sánchez-Camacho et al. 2014). Hay numerosos trabajos sobre la implementación del aula invertida en asignaturas de ciencias de la Tierra (Herrero et al. 2015; 2016; 2019) y otros sobre la aplicación de nuevas tecnologías en la docencia de este campo (Herrero et al. 2016-2; 2018; 2020; Sánchez et al. 2020).

El aprendizaje colaborativo es aquel que se consigue mediante el trabajo en grupo o equipo, siendo la colaboración entre los miembros es un proceso colectivo desde el inicio, donde todos intervienen en la realización de las tareas y donde la diferenciación de roles dentro de los equipos surge espontáneamente en la dinámica interactiva (Roselli, 2016). El aprendizaje basado en proyectos forma parte del llamado "aprendizaje activo" y consiste en que los alumnos deben resolver un problema o reto planteado por el profesor, para ello 
tendrán que documentarse, elegir las herramientas, etc. (Álvarez-Herrero, 2018). La literatura sobre la aplicación de estas dos metodologías adaptadas a las ciencias de la Tierra es escasa (Fernández-Lozano y Gutiérrez-Alonso, 2016, López Saucedo et al., 2019).

Este trabajo describe la elaboración y creación de material docente (microvideos) para el aula invertida de las asignaturas del Departamento de Ingeniería y Morfología del Terreno de la Universidad Politécnica de Madrid. Este nuevo material ha sido creado por los propios alumnos siguiendo metodologías de aprendizaje basado en proyectos y aprendizaje colaborativo. De esta forma, mediante estrategias innovadoras se ha generado material para el aprendizaje autónomo de los alumnos que se emplea como apoyo de la docencia en el aula invertida. Con esta estrategia se ha podido cerrar el círculo: los alumnos aprenden realizando el material que será posteriormente usado por otros alumnos para aprender.

\section{CONTEXTO}

En las asignaturas de Ciencias de la Tierra de la E.T.S. de Ingenieros de Caminos, Canales y Puertos de la UPM se realizan diferentes ejercicios prácticos sobre interpretación de cortes geológicos y de estabilidad de taludes para los que hay que saber cómo resolver de forma gráfica una serie de ejercicios básicos. Gracias a la combinación de varios de estos ejercicios se puede llegar a resolver problemas complejos de Ciencias de la Tierra aplicadas a la ingeniería civil.

Antes del COVID-19 estos problemas fundamentales eran explicados de forma gráfica en clase por los profesores, con apoyo de la pizarra y diapositivas. Tras la explicación los alumnos debían resolver algunos ejercicios que eran evaluados para comprobar si los habían comprendido.

Normalmente, estos rudimentos exigían numerosas tutorías posteriores a alumnos que no terminaban de entender algunos de los problemas en clase, o que por diversos motivos no habían podido asistir. Fue entonces cuando surgió la idea de realizar una serie de videotutoriales que pudieran estar a disposición de los alumnos para que pudieran consultarlos cuantas veces lo necesitaran para aprender los ejercicios básicos.

Debido a la pandemia, las clases del segundo semestre del curso 19-20 se han realizado de forma virtual y las del curso 2021 han sido semi-presenciales. Esto dificultó enormemente las explicaciones gráficas y su comprensión por parte del alumnado, haciendo más necesario aun la elaboración de material de apoyo.

El objetivo de los microvideos es ayudar a los estudiantes de varias asignaturas relacionadas con la geología y la ingeniería del terreno en el Grado de Ingeniería Civil y Territorial, además de en asignaturas del grado en Geología de la UCM. Inicialmente, unos 300 alumnos al año podrán beneficiarse del material generado.

\section{DESCRIPCIÓN}

La primera tarea realizada por los miembros del equipo fue la identificación de los ejercicios básicos que se iban a proponer a los alumnos. Al ser un proyecto cuyo resultado debía ser una colección de videos cortos, de entre 3 y 5 minutos (microlearning), uno de los criterios para su selección fue que fueran problemas sencillos que se pudieran resolver de forma gráfica en pocos minutos. El otro criterio que entró en la selección fue la importancia de esos problemas básicos para después poder resolver problemas más complejos. Una vez seleccionados los ejercicios a resolver se hizo un llamamiento a los alumnos de la asignatura de Geología Aplicada a las Obras Públicas (un primer llamamiento a los alumnos del curso 19-20 y al año siguiente a los del curso 20-21). Para este trabajo los equipos fueron propuestos por los propios alumnos, asignando los profesores a los equipos menos numerosos a los alumnos que quisieron participar pero no tenían grupo. Cada grupo debía realizar un microvideo explicando cómo se resolvería (a modo de videotutorial) un problema determinado.

Una vez asignados los problemas a los equipos de trabajo se realizó una reunión inicial (Kick-off meeting), virtual para los grupos del curso 19-20 y presencial para los del 20-21, con cada equipo en el que se fijaron los objetivos, entregables, etc. Cada grupo ha participado en todas las fases del proyecto (realización del guion del video, grabación del mismo $\mathrm{y}$, aquellos grupos interesados, participación en el montaje y edición de su video). Cada video consta de una pequeña introducción en la que se plantea el problema, una resolución detallada con un juego de datos y otra resolución adicional con otros datos, más rápida y sin explicación, para que los alumnos que vean el video, puedan ver otro ejemplo.

Los alumnos interesados en participar se dividieron en grupos de 5-6 personas. Pese a ser una actividad voluntaria en el curso 2019-2020 se ofrecieron unos 150 alumnos, el $80 \%$ de los alumnos matriculados. En el curso 2020-2021 se ofrecieron sólo los videos relacionados con cuestiones que no se habían incluido el año anterior o que por calidad no pudieron usarse, habiéndose apuntado unos 30 alumnos. Lo primero que hicieron los grupos fue el guion del microvideo que fue revisado por los profesores, sugiriendo los cambios necesarios para su mejora. Una vez aprobado el borrador por los profesores, los alumnos procedieron a grabar el microvideo. Los alumnos del curso 1920 en casa, con sus medios (normalmente un móvil situado en posición cenital), mientras que los grupos del curso 20-21 realizaron la grabación en las instalaciones del Gabinete de Tele-educación de la UPM (Fig. 1). Cada equipo grabó sus videos en el formato que ellos eligieron, algunos todo el video en una toma continua, otros en varios fragmentos de video; por ejemplo uno por cada sección, unos grabaron el sonido a la vez que el video y otros lo grabaron en una pista de sonido independiente. 


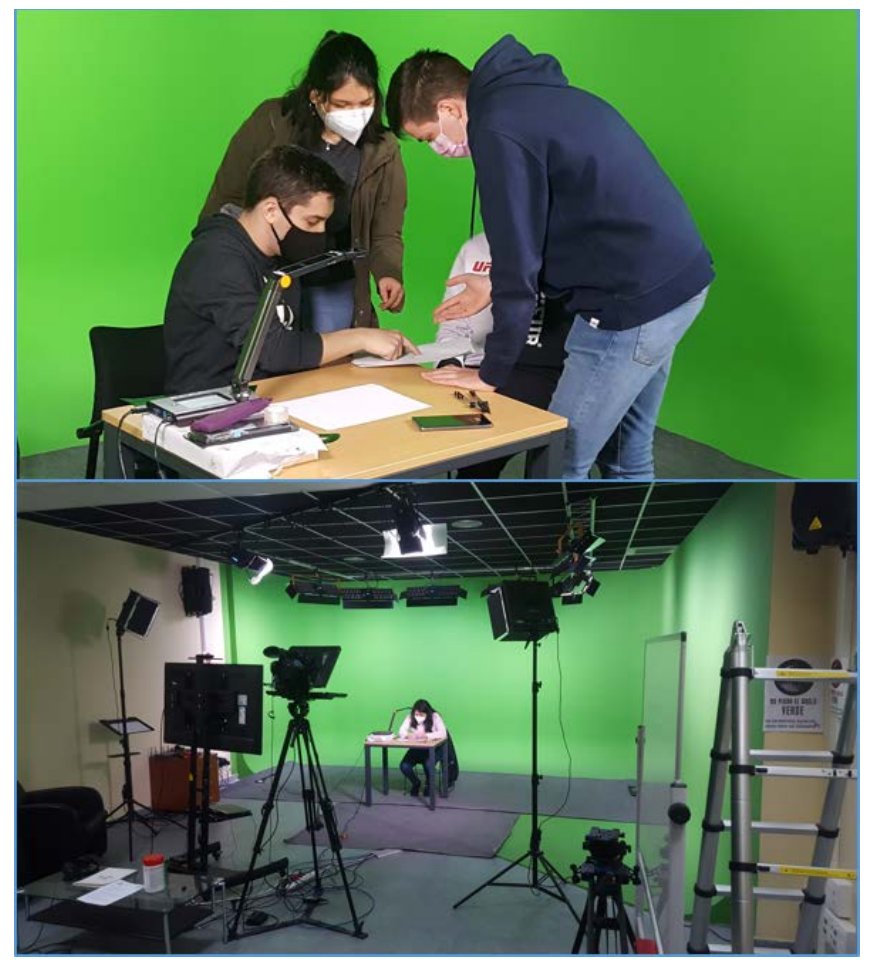

Fig. 1. Preparación y grabación de los videos con cámara cenital

Con ese material en bruto y mediante el programa Camtasia, los videos se montaron y editaron añadiendo a cada uno una página de inicio o portada, una mosca (marca de agua) con el escudo de la Universidad y una página de cierre o contraportada. En la portada se incluye información sobre el problema que enseña a resolver cada video y los integrantes del equipo (Fig. 2). En la contraportada se nombran los miembros del Proyecto de Innovación Educativa y las restricciones de uso del material (Fig. 2).

En la realización se ha cuidado especialmente la calidad de la imagen y la buena visibilidad de lo que se dibuja y escribe ya que una gran parte de los alumnos que van a consultar el video lo harán a través de teléfonos móviles o tablets con tamaños de pantalla reducidos (Fig. 3). En el sonido se ha evitado poner música de fondo para no entorpecer la voz del narrador que va explicando el proceso de resolución del ejercicio.

Los videos se han creado en formato .mp4 y se han subido a la nube corporativa de la Universidad Politécnica de Madrid. Los enlaces a los videos se han incluido en una sección específica de herramientas para el autoaprendizaje en el Moodle de las diferentes asignaturas.
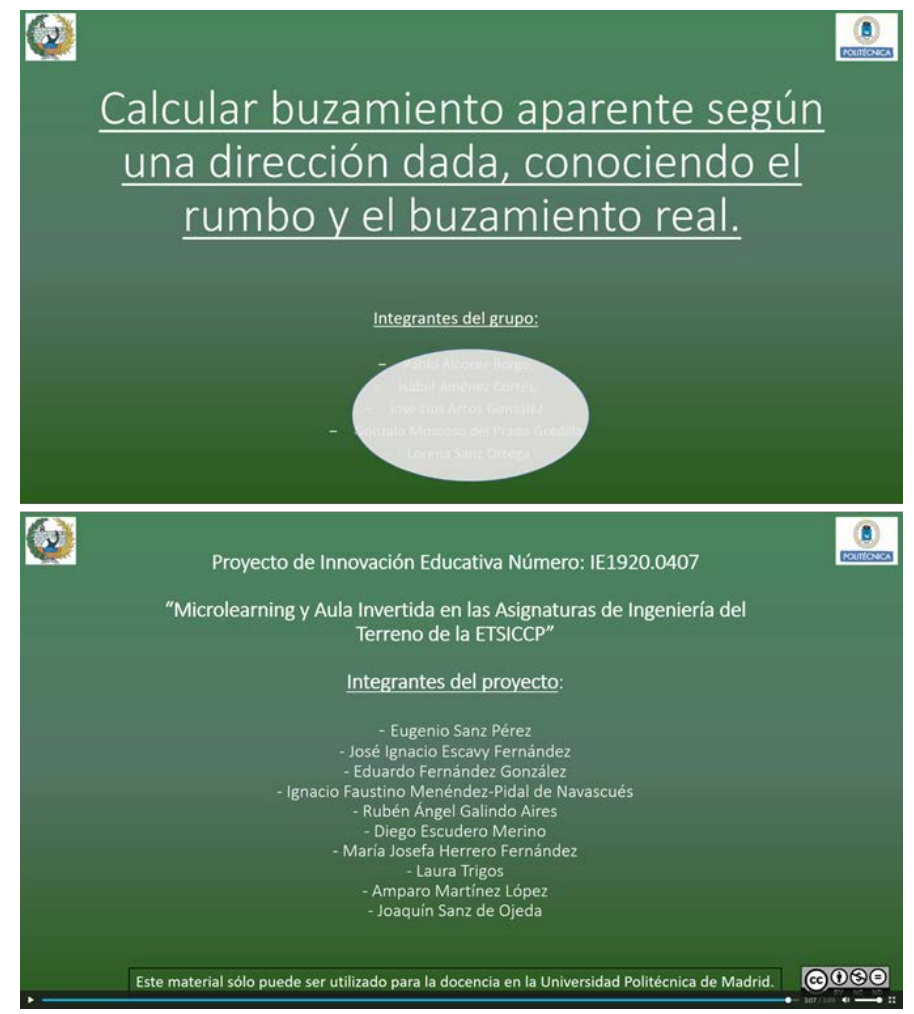

Fig. 2.- Portada (arriba) y contraportada (abajo) de uno de los videos realizados.

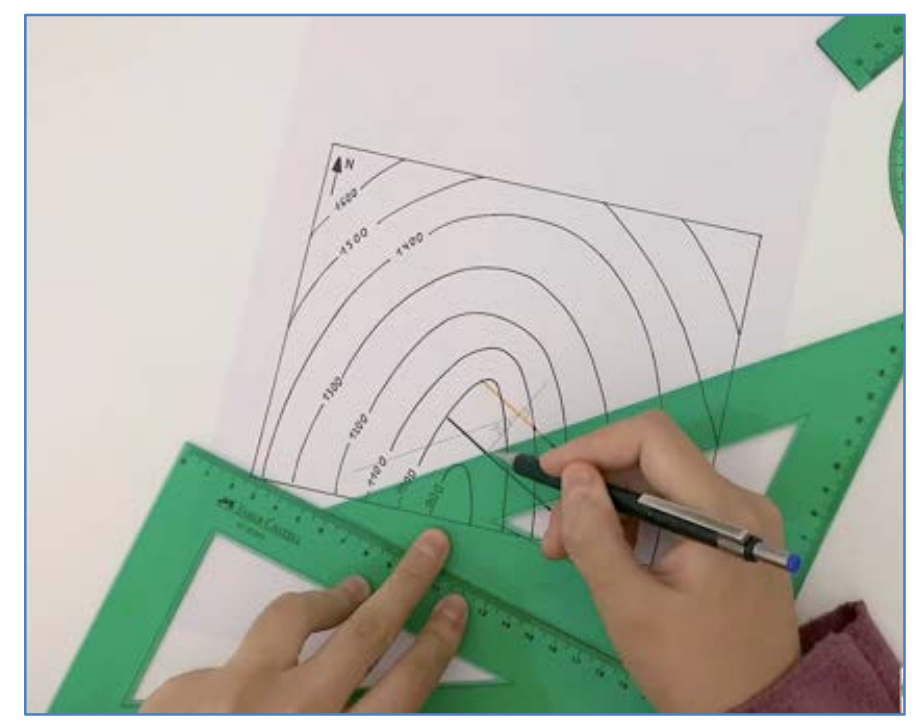

Fig. 3.- Fotograma de uno de los videos realizados por los alumnos

\section{Resultados}

De todos los videos realizados se han seleccionado los 20 con mejor calidad para ponerlos a disposición de los alumnos a través de la plataforma Moodle. Con el fin de evaluar el impacto que generan en el aprendizaje, se han realizado encuestas a los estudiantes que han hecho los videos y a los estudiantes que no han participado en los videos pero que los han usado como herramienta para el autoaprendizaje. 
Las encuestas a los alumnos que han realizado los videos tenían las siguientes preguntas, que debían valorar de 1 a 5 siendo 1 "totalmente en desacuerdo" y 5 "totalmente de acuerdo":

1.- Consideras que esta actividad te ha ayudado a aprender a resolver cortes geológicos

2.- Consideras que esta actividad te ha ayudado a aprender otras competencias transversales (ej. trabajo en equipo, diseño, grabación y edición de videos, etc.)

3.- Crees que los videos realizados van a ayudar a aprender a los alumnos que los vean en el futuro

4.- Crees que el formato elegido (microvideo) es el adecuado para este tipo de aprendizaje

5.- Piensas que el tiempo empleado en esta actividad ha merecido la pena viendo el resultado

Un total de 84 alumnos han respondido la encuesta. Los resultados se muestran en la Tabla 1 .

Tabla 1.- Resultados de la encuesta a los alumnos que han participado en la realización de los videos

\begin{tabular}{|l|c|c|c|c|c|c|}
\cline { 2 - 7 } \multicolumn{1}{c|}{} & \multicolumn{5}{c|}{ Puntuación } & Media \\
\cline { 2 - 7 } \multicolumn{1}{c|}{} & $\mathbf{1}$ & $\mathbf{2}$ & $\mathbf{3}$ & $\mathbf{4}$ & $\mathbf{5}$ & Ponderada \\
\hline Pregunta 1 & 0 & 0 & 3 & 28 & 53 & 4.6 \\
\hline Pregunta 2 & 0 & 0 & 0 & 19 & 65 & 4.8 \\
\hline Pregunta 3 & 0 & 0 & 0 & 0 & 84 & 5.0 \\
\hline Pregunta 4 & 0 & 0 & 22 & 33 & 29 & 4.1 \\
\hline Pregunta 5 & 0 & 6 & 21 & 38 & 19 & 3.8 \\
\hline
\end{tabular}

Todos los estudiantes creen que el material creado va a ayudar a aprender a futuros alumnos. Una gran mayoría piensa que ha sido útil para aprender sobre el tema propuesto y otras competencias transversales. Las respuestas sobre el formato de los videos y sobre el tiempo empleado en su realización han sido más variables aunque la mayoría tiene una opinión favorable o muy favorable.

Los videos se han puesto a disposición de los alumnos del curso 20-21 una vez terminados y se ha lanzado una encuesta a los estudiantes que los han usado y que no han estado involucrados en su realización. Un total de 23 alumnos han respondido el cuestionario. Las preguntas a las que han tenido que responder han sido:

1.- Crees que este material es útil para el aprendizaje de cortes geológicos

2.- Crees que el formato de microvideo es el adecuado para este tipo de material de autoaprendizaje

3.- Recomendarías el empleo de este material a tus compañeros de clase

Las respuestas obtenidas se muestran en la Tabla 2. La totalidad de los alumnos que han respondido la encuesta piensan que el material es muy útil y que el formato es el adecuado, mientras que una inmensa mayoría recomendaría este material para el autoaprendizaje de sus compañeros.
Tabla 2.- Resultados de la encuesta a los alumnos que han usado los videos para su aprendizaje.

\begin{tabular}{|l|c|c|c|c|c|c|}
\cline { 2 - 7 } \multicolumn{1}{c|}{} & \multicolumn{5}{c|}{ Puntuación } & Media \\
\cline { 2 - 7 } \multicolumn{1}{c|}{} & $\mathbf{1}$ & $\mathbf{2}$ & $\mathbf{3}$ & $\mathbf{4}$ & $\mathbf{5}$ & Ponderada \\
\hline Pregunta 1 & 0 & 0 & 0 & 0 & 23 & 5.0 \\
\hline Pregunta 2 & 0 & 0 & 0 & 0 & 23 & 5.0 \\
\hline Pregunta 3 & 0 & 0 & 0 & 2 & 21 & 4.9 \\
\hline
\end{tabular}

En general, tanto los resultados obtenidos de los alumnos que han generado el material como de los que lo están utilizando demuestran un alto grado de satisfacción y lo consideran una buena herramienta para el autoaprendizaje.

\section{CONCLUSIONES}

La combinación de diferentes estrategias de innovación docente (aprendizaje colaborativo y aprendizaje basado en proyectos) para la creación de material docente, supone un avance al maximizar el aprendizaje tanto de los que realizan el material como de los futuros usuarios para autoaprendizaje en el contexto del aula invertida. No solo adquieren competencias relativas a las asignaturas sino que además aprenden a trabajar en equipo y otras habilidades como la elaboración de material $\mathrm{y}$ el uso de programas de generación y edición de video. La participación directa de los alumnos en la generación de material docente les aporta además seguridad en la forma de trabajar y les genera sensación de contribuir con su trabajo a una actividad formativa de interés para ellos mismos.

Esta experiencia de creación de microvideos por parte de grupos de alumnos ha sido valorada como muy positiva tanto por los alumnos que han realizado el material como por los usuarios finales.

Este tipo de actividad puede ser aplicada en un gran número de contextos en los que los problemas se puedan fraccionar en pequeñas partes que puedan ser resueltas en pocos minutos.

\section{AGRADECIMIENTOS}

Este proyecto de innovación educativa se ha podido llevar a cabo gracias a la convocatoria de Proyectos de Innovación Educativa de la Universidad Politécnica de Madrid en la que obtuvo la financiación necesaria en la convocatoria de 2019 (IE1920.0407). Nuestro más sincero agradecimiento al Gabinete de Tele-Educación (GATE) de la UPM y en especial al personal del departamento de audiovisuales por toda su ayuda.

\section{REFERENCIAS}

Álvarez-Herrero, J.F. (2018). El aprendizaje basado en proyectos (ABP). Informe ODITE sobre Tendencias Educativas 1, 14-19

Domínguez LC, Vega NV, Espitia EL, Sanabria AE, Corso C, Serna AM y Osorio C. (2015). Impacto de la estrategia de aula invertida en el ambiente de aprendizaje en cirugía: una comparación con la clase magistral. Biomédica 35(5):13-21

Fernández-Lozano, J y Gutiérrez-Alonso, G. (2016). Aula 3.0: Una nueva forma de aprender geología. El uso de las apps Trnio ${ }^{\circledR}$ y Skechfab ${ }^{\circledR}$ para construir modelos $3 \mathrm{D}$ con el 
móvil. Enseñanza de las Ciencias de la Tierra, 24(2): 163168

Herrero, M.J., Arribas, M.E., Arribas, J., Escavy, López Acevedo, F. (2015). Creación de Aulas Inversas (Flipped Classroom) en prácticas de campo en Petrología Sedimentaria. Memoria PIMCD 2014. https:/eprints.ucm.es/id/eprint/28666/

Herrero, M.J., Arribas, M.E., Arribas, J., Alvarez Sierra, M.A., Escavy, J.I., Ureta, M.S., López Acevedo, F. (2016). Creación de rutas geológicas como recursos docentes (Flipped Classroom) en las asignaturas de Petrología Sedimentaria y Paleontología Aplicada. Memoria PIMCD 2015. https://eprints.ucm.es/id/eprint/35464/

Herrero, M.J., Álvarez, M.A., Ureta, M.S., Castiñeiras, P., Arribas, J., Escavy, J.I., López Acevedo, F. (2017). Rutas geológicas virtuales como recurso educativo abierto (open access) en asignaturas de Geología Aplicada. Memoria PIMCD 2017. https://eprints.ucm.es/id/eprint/43617/1/memoria 202016-17 20Proyectos 20Innovación.pdf

Herrero, M.J., Álvarez, M.Á., Arribas, J., Arribas, M.E., Castiñeiras, P., Escavy, J.I., Insúa, J.M., Trigos, L., López Acevedo, F.J., Ureta, S. y Uribelarrea, D. (2018). Uso de tecnologías emergentes para la elaboración de salidas de campo virtuales para asignaturas de Ciencias de la Tierra. Memoria PIND https://eprints.ucm.es/id/eprint/48218/

Herrero Fernández, M. J., Escavy Fernández, J. I., Insúa Arévalo, J. M., Horra del Barco, R de la., Sánchez Moya, Y., Sopeña Ortega, A., Álvarez Gómez, J. A., López Acevedo, F. J., Jiménez Molina, D., Trigos Luque, L., Fregenal Martínez, M. A., Martínez Díaz, J. J., Menéndez-Pidal de Navascués, I., Sanz Pérez, E., Varas Muriel, M. J., Sanz de Ojeda, J. 2021. "Innovación en contenidos virtuales vía tecnologías digitales: Modelos Geológicos 3D y Salidas de campo virtuales en asignaturas de Ciencias de la Tierra. Memoria Proyecto de Innovación Docente 2020-157. E-prints UCM es

Herrero, M.J., Escavy, J.I., Insúa, J.M., de la Horra, R., Sánchez Moya, Y., Sopeña, A., Álvarez Gómez, J.A., López Acevedo, F.J., Jiménez, D., Trigos, L., Fregenal, M.A., Martínez, J.J., Menéndez-Pidal, I., Rey, J.J., Sanz Pérez, E., Varas, M.J., Sanz de Ojeda, J., Sanz de Ojeda, P., Arribas, J., Álvarez, M.Á. y Uribelarrea, D., (2019). RPAS (Remotely Piloted Aircraft Systems) para la elaboración de salidas de campo virtuales como recursos docentes "flipped classroom" para Grados relacionados con Ciencias de la Tierra. Memoria PIND 2019. https:/eprints.ucm.es/id/eprint/57600/

Herrero, M.J., Escavy, J.I., Insúa, J.M., de la Horra, R., Sánchez Moya, Y., Sopeña, A., Álvarez, JA., López Acevedo, F.J., Jiménez, D., Trigos, L., Fregenal, M.A., Martínez, J.J., Menéndez-Pidal, I., Sanz, E., Varas, M.J., Sanz de Ojeda, J. (2021). Innovación en contenidos virtuales vía tecnologías digitales: Modelos Geológicos 3D y Salidas de campo virtuales en asignaturas de Ciencias de la Tierra. Memoria Proyecto de Innovación Docente 2020-157. Eprints UCM_es

Sánchez Moya, Y., Jiménez, D., Álvarez, J.A., Martínez, J.J., Insúa, J.M., Fregenal, M.A., Herrero, M.J., Varas, M.J., Morellón, M., de la Horra, R., Sanz, E., Escavy, J.I., Sopeña, A., Menéndez-Pidal, I., Trigos, L., Rey, J.J., López Acevedo, F.J., Sanz De Ojeda, P. y Sanz De Ojeda, J. (2020). Uso de RPAS (Remotely Piloted Aircraft System) para la docencia y divulgación de las Ciencias de la Tierra. Memoria PIMCD 2019. https:/eprints.ucm.es/id/eprint/61148/

López-Saucedo, F., Batista-Rodríguez, J., Monsiváis-Durón, D., Almaguer-Carmenates, Y., Hernández-Rosales, A., Hernández-Rodríguez, J. (2019). El aprendizaje colaborativo y las Ciencias de la Tierra en la asignatura de Probabilidad y Estadística. Revista Internacional de Investigación e Innovación Tecnológica 39, 1:16

Martínez-Olvera, W., Esquivel-Gámez, I. y Martínez, J. (2014). Aula Invertida o Modelo Invertido de Aprendizaje: Origen, Sustento e Implicaciones. En Los Modelos Tecno-Educativos, revolucionando el aprendizaje del siglo XXI. Mexico D.F., 256pp

Moreno-Correa, S. (2020). La innovación educativa en los tiempos del Coronavirus. Salutem Scientia Spiritus 2020; $6(1): 14-26$

Roselli, N.D. (2016). El aprendizaje colaborativo: Bases teóricas y estrategias aplicables en la enseñanza universitaria. Propósitos y Representaciones, 4 (1): 219280. http://dx.doi.org/10.20511/pyr2016.v4n1.90

Sánchez-Camacho C, Azpeleta C, Gal B, Suárez F. (2014). Flipped Classroom como herramienta para la integración de contenidos en asignaturas básicas de la titulación de medicina. XI Jornadas Internacionales de Innovación Universitaria. 189-96. 